EUROPHYSICS LETTERS

16 January 1998

Europhys. Lett., (), pp. (1998)

\title{
$\alpha-{ }^{5} \mathrm{He}$ decaying states and the ground state rotational band of ${ }^{9} \mathrm{Be}$
}

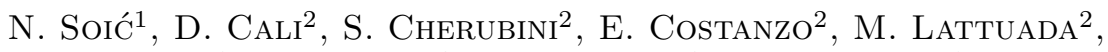 \\ Đ. Miljanić ${ }^{1}$, S. Romano ${ }^{2}$, C. Spitaleri ${ }^{2}$ and M. Zadro ${ }^{1}$ \\ 1 Ruđ̈er Bošković Institute, Zagreb, Croatia \\ 2 INFN-Laboratorio Nazionale del Sud and Università di Catania, Catania, Italy \\ (received ; accepted in final form ) \\ PACS. 25.70-z - Low and intermediate energy heavy-ion reactions. \\ PACS. $27.20+\mathrm{n}-6 \leq A \leq 19$.
}

\begin{abstract}
In a measurement of the ${ }^{9} \mathrm{Be}\left({ }^{7} \mathrm{Li}, \alpha^{7} \mathrm{Li}\right) \alpha$ n reaction at $E_{\mathrm{i}}=52 \mathrm{MeV}$ it is unambigously established for the first time that the ${ }^{9}$ Be excited states around 6.5 and $11.3 \mathrm{MeV}$ decay into the $\alpha+{ }^{5} \mathrm{He}$ channel. This fact may support previous claims that the $11.3 \mathrm{MeV}$ state is also a member of the ground state rotational band.
\end{abstract}

Introduction. $-{ }^{9} \mathrm{Be}$ nucleus has one of the largest deformations among the stable nuclei. Beside the ground state $\left(\frac{3}{2}^{-}\right)$only two other members $\left(\frac{5}{2}^{-}, \frac{7}{2}^{-}\right)$of the $K=\frac{3}{2}^{-}$rotational band are well established. However, there is a standing controversy about the fourth member of the band. A state from the doublet with the excitation energies between 11 and $12 \mathrm{MeV}$ has been sometimes mentioned as possible $\frac{9}{2}^{-}$member (see $e$. g. 任). A bump is visible at these excitations in energy spectra from the $\alpha$ - particle inelastic scattering (strictly isoscalar excitation [2, 3, 田, 5]). Peterson [3] even measured the angular distribution for the 11.28 $\mathrm{MeV}$ state and concluded that it should be the expected $\frac{9}{2}^{-}$state. However, recent results on electron and proton inelastic scattering [6, 7 were interpreted in another way:

i) The level, listed in recent compilation [8] at $6.76 \mathrm{MeV}$, was separated into two components identified as the $\frac{7}{2}^{-}$member of the band located at $6.38 \mathrm{MeV}$ and the $\frac{9}{2}^{+}$weak coupling state at $6.76 \mathrm{MeV}$; ii) In the case of the $11 \mathrm{MeV}$ states it was claimed that the (p,p') data did not give any and $\left(\mathrm{e}, \mathrm{e}^{\prime}\right)$ data only a weak evidence of the state at $11.81 \mathrm{MeV}$. For the other state at $11.28 \mathrm{MeV} J^{\pi}=\frac{9}{2}^{+}$was given as the most likely assignment. However, from the delayed $\alpha-$ particle spectra of the ${ }^{9} \mathrm{Li} \beta$ - decay it was concluded that the $11.28 \mathrm{MeV}$ state was a negative parity state, probably $\frac{3}{2}^{-}$[9].

Recently, the microscopic multicluster model [10] was applied to the study of the mirror nuclei, ${ }^{9} \mathrm{Be}$ and ${ }^{9} \mathrm{~B}$. They were described in a three - cluster model comprising two $\alpha$ - particles and a single nucleon. From this calculation it was concluded that the ${ }^{5} \mathrm{He}+\alpha$ configuration 
is a very good approximation for the ${ }^{9} \mathrm{Be}$ ground state wave function. There have also been many studies on the $\alpha-\alpha$ quasi-free scattering on ${ }^{9} \mathrm{Be}$ 11]. They all have found large $\alpha$ - particle spectroscopic factors for the ground state. In the analysis of the data from these studies it has been assumed that the knocked out cluster is bound in a $3 S$ or $2 D$ state with equal occupation probability. It was also claimed that some quasi-free reactions on the ${ }^{5} \mathrm{He}$ cluster in ${ }^{9} \mathrm{Be}$ were observed, like $\left({ }^{3} \mathrm{He}, \mathrm{t}\right)$ and $\left({ }^{3} \mathrm{He}, \alpha\right)$ reactions [12, 13].

All these results point out that one could expect that the unbound states of the rotational band having similar structure to the ground state could preferentially decay into the $\alpha-{ }^{5} \mathrm{He}$ channel. In the most recent compilation of ${ }^{9} \mathrm{Be}$ states $[8]$ there is no mention of $\alpha$ - decay from states between 6 and $7 \mathrm{MeV}$ as well as between 11 and $12 \mathrm{MeV}$. From ${ }^{9} \mathrm{Li}\left({ }^{9} \mathrm{C}\right) \beta$ - decay studies 14, 15 there have been claims that some states in ${ }^{9} \mathrm{Be}\left({ }^{9} \mathrm{~B}\right)$ decay into the $\alpha+{ }^{5} \mathrm{He}$ $\left(\alpha+{ }^{5} \mathrm{Li}\right)$ channel. In this way Nyman et. al. [14] could explain the high energy part of the $\beta$ - delayed $\alpha$ - particle spectra either by the decay of the $11.81 \mathrm{MeV}$ state alone or by addition of $30 \%$ contribution of the $11.28 \mathrm{MeV}$ state. This is in contrast to the explanation of the same spectra given by Langevin et. al. [9], who invoke simultaneous breakup of the 11.28 $\mathrm{MeV}$ state into $2 \alpha+\mathrm{n}$. Both papers did not explore the third possibility of decay of the ${ }^{9} \mathrm{Be}$ $11 \mathrm{MeV}$ state(s): the neutron emission into the low energy tail of the very broad $11.4 \mathrm{MeV}$ state of ${ }^{8} \mathrm{Be}$. Because of all this one cannot claim unequivocally from the ${ }^{9} \mathrm{Li}$ data that ${ }^{9} \mathrm{Be}$ $11 \mathrm{MeV}$ states decay into the $\alpha+{ }^{5} \mathrm{He}$ channel. In order to learn more about these states and their $\alpha-{ }^{5} \mathrm{He}$ decay one should perform kinematically complete measurements of the processes involving these states.

Experiment. - Recently, an experiment has been performed to study different ${ }^{9} \mathrm{Be}+{ }^{7} \mathrm{Li}$ reactions [16]. In the experiment a $52 \mathrm{MeV}^{7} \mathrm{Li}$ beam from the SMP Tandem Van de Graaff accelerator (Laboratorio Nazionale del Sud) was used to bombard a self-supported beryllium target $\left(400 \mu \mathrm{g} \mathrm{cm}^{-2}\right)$. The outgoing charged particles were detected and identified in several particle telescopes consisting either of silicon surface barrier ( $\Delta E$ and $E$ ) detectors (SDT) or of ionization chamber and position sensitive silicon detector (ICPSDT). Coincidence events between any two telescopes were recorded. At the beginning of the experiment single telescope spectra were recorded. Only a part of the data is discussed here.

Results and discussion. - A typical $Q$ - value spectrum for the ${ }^{9} \mathrm{Be}\left({ }^{7} \mathrm{Li},{ }^{7} \mathrm{Li}\right){ }^{9} \mathrm{Be}$ reaction is shown on fig. 1 . The spectrum was obtained from the ${ }^{7} \mathrm{Li}$ data measured with the ICPSDT positioned at $24^{\circ}$ with an opening angle of $4^{\circ}$. Four distinct groups are observed corresponding to ${ }^{9} \mathrm{Be}$ excitation energies of $0.0,2.4,6.4$ and $11.3 \mathrm{MeV}$. The satellites clearly seen for the first two groups correspond to the simultaneous excitation of the ${ }^{7} \mathrm{Li}$ projectile to its first excited state $(478 \mathrm{keV})$. Similarly to the spectra from $\alpha$ - particle scattering on ${ }^{9} \mathrm{Be}$ [2, 3, 4, 4], the ${ }^{7} \mathrm{Li}$ spectra show strong excitation of the states with energies approximately following the $C$ $\left[J(J+1)-J_{0}\left(J_{0}+1\right)\right]$ rule $\left(C\right.$ is a constant and $J, J_{0}$ the spins of excited and ground state, respectively).

In the analysis of the $\alpha-{ }^{7} \mathrm{Li}$ coincidence data one could notice that a large part of the yield from the ${ }^{9} \mathrm{Be}\left({ }^{7} \mathrm{Li},{ }^{7} \mathrm{Li} \alpha\right) \alpha$ n reaction is concentrated on the kinematical loci corresponding to the ${ }^{5} \mathrm{He}$ ground state $(Q=-2.5 \mathrm{MeV})$. This is illustrated on fig. 2 . by the $Q$ - value spectra obtained from the ${ }^{7} \mathrm{Li}-\alpha$ coincidence data measured at the detectors angle pair $24^{\circ}-50^{\circ}$. The full line represents the spectrum with all the data taken into account and the dashed line is the one with only the data having $\alpha$ - particle energies above $7 \mathrm{MeV}$ i.e. excluding the contribution of the strongly excited $2.43 \mathrm{MeV}$ state of ${ }^{9} \mathrm{Be}$, which decays into the $\mathrm{n}+{ }^{8} \mathrm{Be}$ channel. One can also notice a broad structure around $Q=-6 \mathrm{MeV}$ which may be due to the reactions leading to the first excited state of ${ }^{5} \mathrm{He}$. 
Fig. 3. shows the ${ }^{9} \mathrm{Be}$ excitation energy spectrum obtained from the ${ }^{7} \mathrm{Li}-\alpha$ coincidence data, measured with an ICPSDT at $26^{\circ}$ and a SDT at $50^{\circ}$, with a cut imposed on the $Q$ value spectrum corresponding to the ${ }^{5} \mathrm{He}$ ground state. The higher yield for $E_{\mathrm{x}}$ between 4 and $15 \mathrm{MeV}$ is in part due to the quasi-free ${ }^{7} \mathrm{Li}-\alpha$ scattering contribution. This contribution was also observed for all those angle pairs satisfying kinematical quasi-free scattering condition [16. Two distinct peaks are also visible corresponding to the excitation energies of 6.5 and 11.3 MeV. From the spectra it was not possible to conclude, whether the broad peak centered between 6 and $7 \mathrm{MeV}$ is due to two levels at these excitations as claimed in [6, 7] or to a single broad state [8]. It may be added that three different theoretical calculations [10, 17, 18] predict the $\frac{7}{2}^{-}$and $\frac{9}{2}^{+}$states at these energies to be very close to each other $(\Delta \mathrm{E} \leq 400 \mathrm{keV})$.

In the most recent one [10] the widths were also calculated and the $\frac{9}{2}^{+}$state was a factor of 2.4 wider than the $\frac{7}{2}^{-}$state $(2.9$ and $1.2 \mathrm{MeV})$. However, the same theories also predict several other states of ${ }^{9} \mathrm{Be}$ but their existence has not been confirmed experimentally. For illustration it is also shown the result of a calculation with only two Breit - Wigner terms (multiplied by the phase space factor) with the following excitation energies and widths of these states: $E_{1}^{\text {exc }}=6.76 \mathrm{MeV} \Gamma_{1}=1.540 \mathrm{MeV} E_{2}^{\text {exc }}=11.28 \mathrm{MeV} \Gamma_{2}=0.575 \mathrm{MeV}$ as quoted in [8]. The tails of the peaks are probably due to the excitation of ${ }^{9} \mathrm{Be}$ to these states together with the ${ }^{7} \mathrm{Li}$ excitation to its first excited state as observed also in the ${ }^{7} \mathrm{Li}$ single spectra. Obviously, from these measurements it is not possible to say anything about the spins and parities of the observed levels.

The experimental results unambiguously show for the first time that these states of ${ }^{9} \mathrm{Be}$ decay into ${ }^{5} \mathrm{He}_{\text {g.s. }}+\alpha$, which is also the predominant configuration of its ground state. This fact and their strong excitation in $\alpha$ and ${ }^{7} \mathrm{Li}$ inelastic scatterings together with their energies following the "rotational formula" may support the claims that both of them belong to the ground state rotational band. However, if the structure around $6.5 \mathrm{MeV}$ is due to only one state, the third member of the band, then its larger width with respect to the one of the expected fourth member at $11.3 \mathrm{MeV}$ would seem to contradict these claims. Also, in present data there is no evidence for excitation of possible $\frac{11}{2}$ member which should fall around 17 $\mathrm{MeV}$. Obviously, final conclusion on the higher members of the band should wait for additional experimental and theoretical results.

\section{REFERENCES}

[1] F. C. Barker, Nucl. Phys., 83 (1966) 418.

[2] R. G. Summers-Gill, Phys. Rev., 109 (1958) 1591.

[3] R. J. Peterson, Nucl. Phys., A377 (1982) 41.

[4] M. N. Harakeh, J. van Popta, A. Saha and R. H. Siemssen, Nucl. Phys., A 344 (1980) 15.

[5] Subinit Roy, J. M. Chatterjee, H. Majumdar, S.K. Datta, S. R. Banerjee and S. N. Chintalapudi, Phys. Rev. C, 52 (1995) 1524.

[6] J. P. Glickman, W. Bertozzi, T. N. Buti, S. Dixit, F. W. Hersman, C. E. Hyde-Wright, M. V. Hynes, R. W. Lourie, B. E. Norum, J. J. Kelly, B. L. Berman and D. J. Millener , Phys. Rev. C, 43 (1991) 1740.

[7] S. Dixit, W. Bertozzi, T. N. Buti, J. M. Finn, F. W. Hersman, C. E. Hyde-Wright, M. V. Hynes, M. A. Kovash, B. E. Norum, J. J. Kelly, A. D. Bacher, G. T. Emery, C. C. Foster, W. P. Jones, D. W. Miller, B. L. Berman and D. J. Millener, Phys. Rev. C, 43 (1991) 1758.

[8] F. Ajzenberg-Selove, Nucl. Phys., A 490 (1988) 1.

[9] M. Langevin, C Detraz, D. Guillemaud, F. Naulin, M. Epherre, R. Klapisch, S. K. T. Mark, M. de Saint Simon, C. Thibault and F. Touchard, Nucl. Phys., A366 (1981) 449. 
[10] K. Arai, Y. Ogawa, Y. Suzuki and K. Varga, Phys. Rev. C, 54 (1996) 132.

[11] A. A. Cowley, G. F. Steyn, S. V. Förtsch, J. J. Lawrie, J. V. Pilcher, F. D. Smit and D. M. Whittal, Phys. Rev. C, 50 (1994) 2449 and references therein.

[12] K. Kadija, G. Paić, B. Antolković, A. Djaloeis and J. Bojowald, Phys. Rev. C, 36 (1987) 1269.

[13] M. Lattuada, F.Riggi, C. Spitaleri, D. Vinciguerra, Đ. Miljanić, M. Zadro and J. YAO, Nucl. Phys., A 458 (1986) 493.

[14] G. Nyman, R. E. Azuma, P. G. Hansen, B. Jonson, P. O. Larsson, S. Matisson, A. Richter, K. Riisager, O. Tengblad, K. Wilhelmsen and the ISOlde Collaboration, Nucl. Phys., A 510 (1990) 189.

[15] D. Mikolas, B. A. Brown, W. Benenson, L. H. Harwood, E. Kashy, J. A. Nolen Jr., B. Sherrill, J. Stevenson, J. S. Winfield, Z. Q. Xie and R. Sherr, Phys. Rev. C, 37 (1988) 766.

[16] N. Soić, S. Cherubini, E. Costanzo, M. Lattuada, Đ. Miluanić, S. Romano, C. Spitaleri and M. ZADRO, to be published.

[17] H. Furutani, H. Kanada, T. Kaneko, S. Nagata, H. Nishioka, S. Okabe, S. Saito, T. Sakuda And M. Seya, Prog. Theor. Phys. Suppl., 68 (1980) 193.

[18] V. T. Voronchev, V. I. Kukulin, V.N. Pomerantsev, Kh. D. Razikov and G. G. Ryzhikh, Yad. Fiz., 57 (1994) 1964. 
Here is the figure 1.

Fig. 1. - Q - value spectrum for the ${ }^{9} \mathrm{Be}\left({ }^{7} \mathrm{Li},{ }^{7} \mathrm{Li}\right){ }^{9} \mathrm{Be}$ scattering measured at $\mathrm{E}_{\mathrm{i}}=52 \mathrm{MeV}$ and $22^{\circ} \leq \Theta \leq 26^{\circ}$

Here is the figure 2 .

Fig. 2. - Q - value spectra for the ${ }^{9} \mathrm{Be}\left({ }^{7} \mathrm{Li},{ }^{7} \mathrm{Li} \alpha\right) \alpha$ n reaction at $\mathrm{E}_{\mathrm{i}}=52 \mathrm{MeV}$ for $22.5^{\mathrm{o}} \leq \Theta_{1} \leq$ $31^{\circ}$ and $\Theta_{2}=50^{\circ}, \Delta \Phi=180^{\circ}$ obtained from all the data (full line) and from the data with the contributions from $2.43 \mathrm{MeV}$ state of ${ }^{9} \mathrm{Be}$ excluded (dashed line)

Here is the figure 3 .

Fig. 3. $-{ }^{9} \mathrm{Be}$ excitation energy spectra obtained from the data for the ${ }^{9} \mathrm{Be}\left({ }^{7} \mathrm{Li},{ }^{7} \mathrm{Li} \alpha\right){ }^{5} \mathrm{He} g s$ reaction measured at $52 \mathrm{MeV}$ and $26^{\circ} \leq \Theta_{1} \leq 31^{\circ}$ and $\Theta_{2}=50^{\circ}, \Delta \Phi=180^{\circ}$. The curve represents a calculation with two Breit - Wigner terms. 


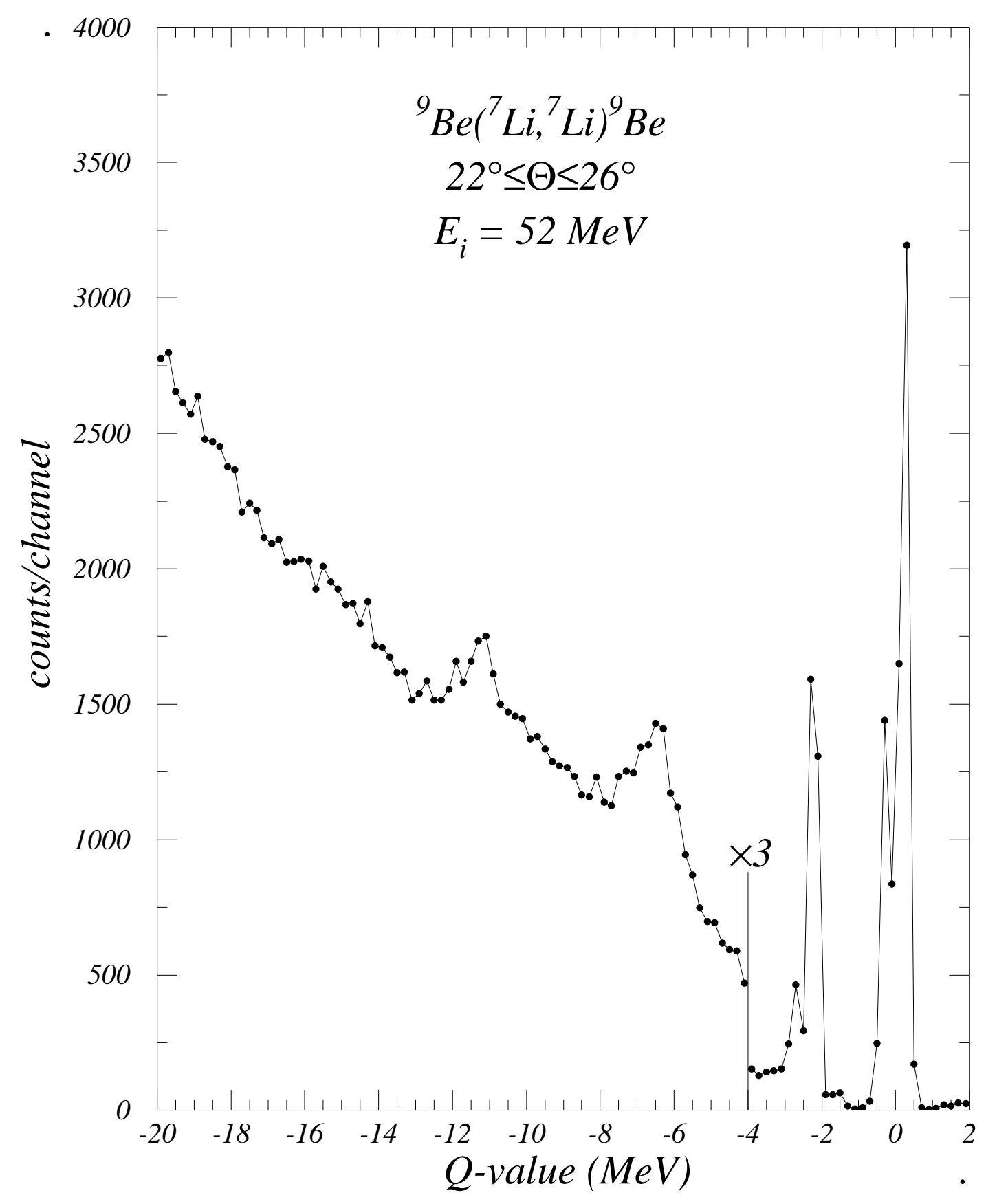




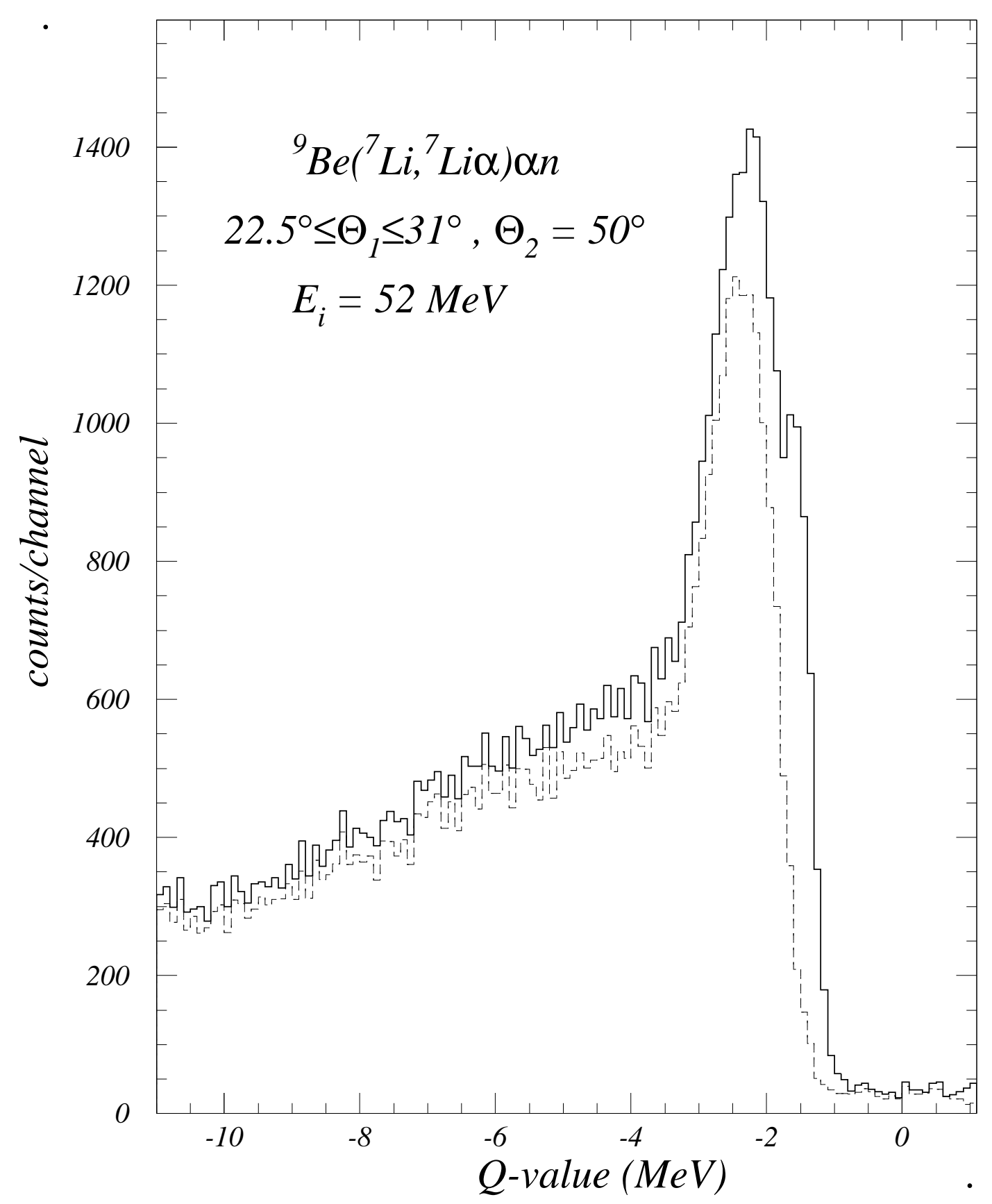




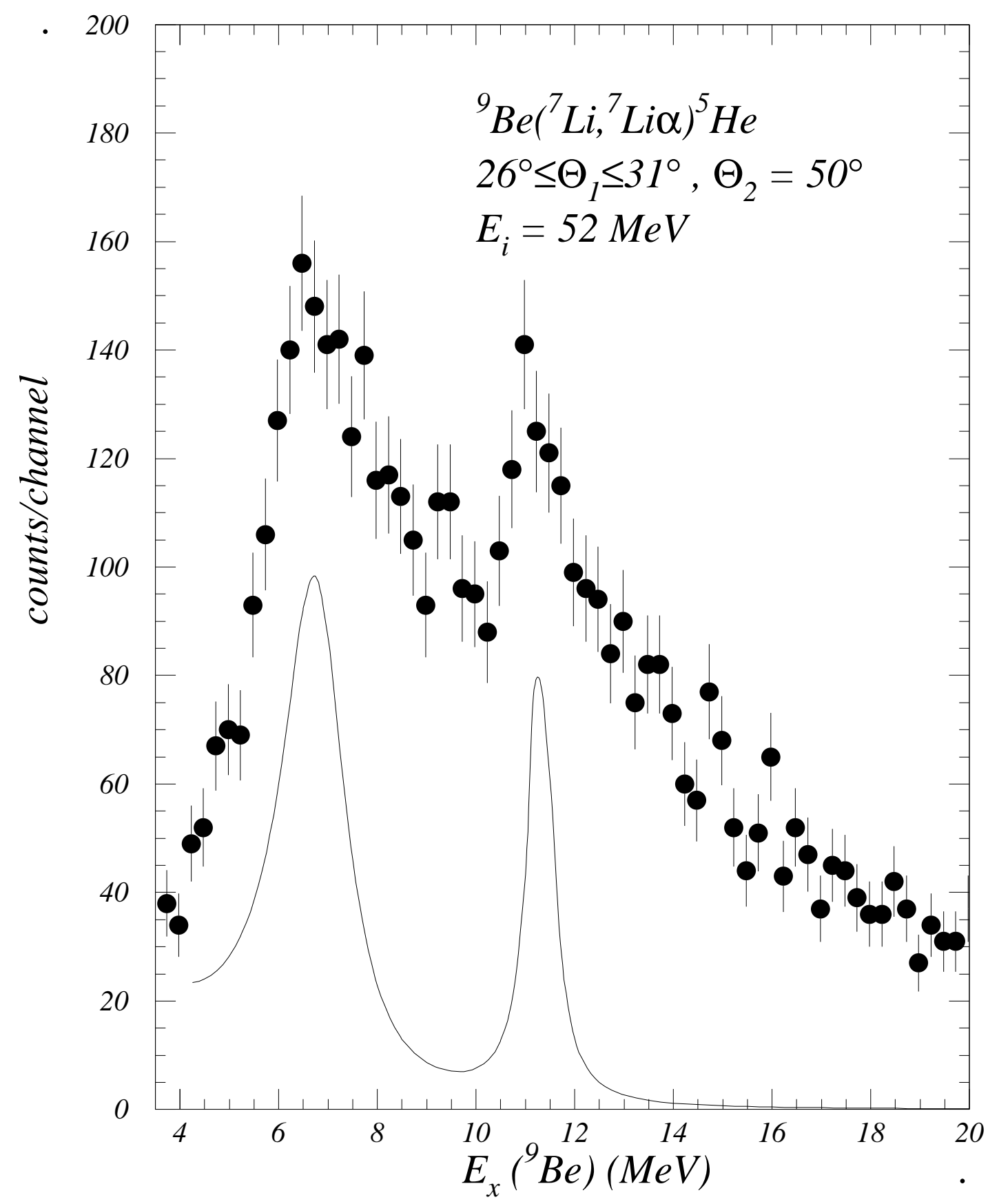

